Mathematical Modelling and Analysis

Volume 19 Number 5, November 2014, 664-675

http://dx.doi.org/10.3846/13926292.2014.980864

(C) Vilnius Gediminas Technical University, 2014
Publisher: Taylor\&Francis and VGTU

http://www.tandfonline.com/TMMA

Print ISSN: 1392-6292

Online ISSN: 1648-3510

\title{
Periodic Solutions of Second Order Impulsive Differential Equations at Resonance via Variational Approach*
}

\author{
Jin $\mathrm{Li}^{a, b}$, Jianlin $\mathrm{Luo}^{a}$ and Zaihong $\mathrm{Wang}^{b}$ \\ ${ }^{a}$ School of Science, Jiujiang University \\ 332005 Jiujiang, China \\ ${ }^{b}$ School of Mathematical Sciences, Capital Normal \\ 100048 Beijing, China \\ E-mail: lijin7912@gmail.com \\ Received February 10, 2014; revised October 6, 2014; published online November 1, 2014
}

Abstract. In this paper, we study the existence of periodic solutions of second order impulsive differential equations atresonance. We prove the existence of periodic solutions under a generalized Landesman-Lazer type condition by using variational method.

Keywords: impulsive differentral equations, Landesman-Lazer type condition, variational method.

AMS Subject Classification: $34 \mathrm{~B} 37$.

\section{Introduction}

We are concerned with periodic boundary value problem of second order impulsive differential equations at resonance

$$
\left\{\begin{array}{l}
x^{\prime \prime}(t)+m^{2} x(t)+f(t, x(t))=e(t), \quad \text { a.e. } t \in[0,2 \pi], \\
x(0)-x(2 \pi)=x^{\prime}(0)-x^{\prime}(2 \pi)=0, \\
x\left(t_{j}^{+}\right)=x\left(t_{j}^{-}\right), \\
\Delta x^{\prime}\left(t_{j}\right):=x^{\prime}\left(t_{j}^{+}\right)-x^{\prime}\left(t_{j}^{-}\right)=I_{j}\left(x\left(t_{j}\right)\right), \quad j=1,2, \ldots, p,
\end{array}\right.
$$

where $m \in \mathbb{N}, f:[0,2 \pi] \times \mathbb{R} \rightarrow \mathbb{R}$ is a Carathéodory function, $e \in L^{1}(0,2 \pi)$, $0<t_{1}<t_{2}<\cdots<t_{p}<2 \pi$, and $I_{j}: \mathbb{R} \rightarrow \mathbb{R}$ is continuous for every $j$.

\footnotetext{
* Research supported by Research Fund for the Doctoral Program of Higher Education of China, No. 11AA0013 and 11401274, Beijing Natural Science Foundation (Existence and multiplicity of periodic solutions in nonlinear oscillations), No. 1112006, the grant of Beijing Education Committee Key Project, No. KZ201310028031, Science and technology landing project of colleges and universities in Jiangxi Province, No. KJLD14092 and Natural Science Foundation Project of Science and Technology Department of Jiangxi province, No. 20132BAB201012 and 20142BDH80027.
} 
When $\Delta x^{\prime}\left(t_{j}\right) \equiv 0$, problem (1.1) becomes the well-known periodic boundary value problem at resonance

$$
\left\{\begin{array}{l}
x^{\prime \prime}(t)+m^{2} x(t)+f(t, x(t))=e(t), \quad \text { a.e. } t \in[0,2 \pi] \\
x(0)-x(2 \pi)=x^{\prime}(0)-x^{\prime}(2 \pi)=0
\end{array}\right.
$$

There are many existence results for problem (1.2) in the literature. Let us mention some pioneering works by Lazer [6], Lazer and Leach [7], and Landesman and Lazer [5]. In [5], a key sufficient condition for the existence of solutions of problem (1.2) is the so-called Landesman-Lazer condition

$$
\begin{aligned}
& \int_{0}^{2 \pi} e(t) \sin (m t+\theta) d t<\int_{0}^{2 \pi}\left[\left(\liminf _{x \rightarrow+\infty} f(t, x)\right) \sin ^{+}(n t+\theta)\right. \\
& \left.-\left(\limsup _{x \rightarrow-\infty} f(t, x)\right) \sin ^{-}(m t+\theta)\right] d t, \forall \theta \in \mathbb{R} \\
& \text { where } \sin ^{ \pm}(m t+\theta)=\max \{ \pm \sin (m t+\theta), 0\} .
\end{aligned}
$$

It is well known that the theory of impulsive differential equations has been recognized to not only be richer than that of differential equations without impulses, but also provide a more adequate mathematicalmodel for numerous processes and phenomena studied in physics, biology, engineering, etc. We refer the reader to the book [4]. Recently, the Dirichlet and periodic boundary conditions problems for second-order differential equations with impulses in the derivative or without impulses are studied by some authors via variational method $[1,2,9,15,17,18,19]$. We also refer to some additional relevant results $[10,12,13,14,16]$. In this paper, we will investigate the problem (1.1) under a more general Landesman-Lazer type condition. Define

$$
F(t, x)=\int_{0}^{x} f(t, s) d s, \quad F_{+}(t)=\liminf _{x \rightarrow+\infty} \frac{F(t, x)}{x}, \quad F_{-}(t)=\limsup _{x \rightarrow-\infty} \frac{F(t, x)}{x} .
$$

Throughout this paper, we give the following fundamental assumptions.

$\left(H_{1}\right)$ There exists $p \in L^{1}([0,2 \pi],[0,+\infty))$ such that $|f(t, x)| \leqslant p(t)$, for a.e. $t \in$ $[0,2 \pi]$ and for all $x \in \mathbb{R}$;

(H2) There exist nonnegative constants $c_{1}, c_{2}, \ldots, c_{p}$ such that for all $s \in \mathbb{R}$,

$$
\left|I_{j}(s)\right| \leqslant c_{j}, \quad j=1,2, \ldots, p
$$

$\left(H_{3}\right)$ For all $\theta \in \mathbb{R}$,

$$
\begin{aligned}
& \sum_{j=1}^{p} c_{j}\left|\sin \left(m t_{j}+\theta\right)\right|+\int_{0}^{2 \pi} e(t) \sin (m t+\theta) d t \\
& \quad<\int_{0}^{2 \pi}\left(F_{+}(t) \sin ^{+}(m t+\theta)-F_{-}(t) \sin ^{-}(m t+\theta)\right) d t
\end{aligned}
$$

We now can state the main theorem of this paper. 
Theorem 1. Assume that the conditions $\left(H_{1}\right),\left(H_{2}\right)$ and $\left(H_{3}\right)$ hold. Then the problem (1.1) has at least one $2 \pi$-periodic solution.

From Theorem 1 we can obtain the following corollary.

Corollary 1. Assume that the conditions $\left(H_{1}\right)$ and $\left(H_{3}^{\prime}\right)$ for all $\theta \in \mathbb{R}$,

$$
\int_{0}^{2 \pi} e(t) \sin (m t+\theta) d t<\int_{0}^{2 \pi}\left(F_{+}(t) \sin ^{+}(m t+\theta)-F_{-}(t) \sin ^{-}(m t+\theta)\right) d t
$$

hold. Then the problem (1.2) has at least one $2 \pi$-periodic solutio Remark 1. By a simple calculation, one can easily derive

$$
\begin{aligned}
& F_{+}(t)=\liminf _{x \rightarrow+\infty} \frac{F(t, x)}{x} \geqslant \liminf _{x \rightarrow+\infty} f(t, x) \\
& F_{-}(t)=\limsup _{x \rightarrow-\infty} \frac{F(t, x)}{x} \leqslant \limsup _{x \rightarrow \infty} f(t, x) .
\end{aligned}
$$

A simple example $f(t, x)=\sin t+\cos x$ illastrates them. Thus condition $\left(H_{3}^{\prime}\right)$ generalizes condition (1.3). Hence, our results improve the related results in the literature mentioned above and some dther results (such as see [10]). It is remarkable that Landesman-Lare condition $\left(H_{3}^{\prime}\right)$ is an "almost" necessary and sufficient condition when $F_{+}$and $F$ are replaced by $f_{+}$and $f_{+}$, where $f_{+}=\lim _{x \rightarrow+\infty} f(t, x), f_{-}=\lim _{x}-\infty f(t, x)$ and $f_{-}(t) \leqslant f(t, x) \leqslant f_{+}(t)$ (see [8, page 70$]$ ). Moreoyer, since we consider the problem with impulses, Theorem 1 is also a complement of the pioneering works.

The rest of the paper is anganized as follows. In Section 2, we shall state some notations, some necessarydefinitions and a saddle theorem due to Rabinowitz. In Section 3, we shall prove Theorem 1.

\section{Preliminarie}

the following, we introduce some notations and some necessary definitions.

$$
H=\left\{x \in H^{1}(0,2 \pi): x(0)=x(2 \pi)\right\}
$$

with the norm

$$
\|x\|=\left(\int_{0}^{2 \pi}\left(x^{\prime 2}+x^{2}\right) d t\right)^{\frac{1}{2}} .
$$

Consider the functional $\varphi(x)$ defined on $H$ by

$$
\begin{aligned}
\varphi(x)= & \frac{1}{2} \int_{0}^{2 \pi} x^{\prime 2}(t) d t-\frac{m^{2}}{2} \int_{0}^{2 \pi} x^{2}(t) d t-\int_{0}^{2 \pi} F(t, x(t)) d t \\
& +\int_{0}^{2 \pi} e(t) x(t) d t+\sum_{j=1}^{p} \int_{0}^{x\left(t_{j}\right)} I_{j}(t) d t .
\end{aligned}
$$


Similarly as in [19], $\varphi(x)$ is continuously differentiable on $H$, and

$$
\begin{aligned}
\varphi^{\prime}(x) v(t)= & \int_{0}^{2 \pi} x^{\prime}(t) v^{\prime}(t) d t-m^{2} \int_{0}^{2 \pi} x(t) v(t) d t-\int_{0}^{2 \pi} f(t, x(t)) v(t) d t \\
& +\int_{0}^{2 \pi} e(t) v(t) d t+\sum_{j=1}^{p} I_{j}\left(x\left(t_{j}\right)\right) v\left(t_{j}\right), \quad \text { for } \forall v(t) \in H .
\end{aligned}
$$

Now, we have the following lemma.

Lemma 1. If $x \in H$ is a critical point of $\varphi$, then $x$ is a $2 \pi$-periodic solution of Eq. (1.1).

The proof of Lemma 1 is similar as Lemma 2.1 in [2], so we

We say that $\varphi$ satisfies (PS) if every sequence $\left(x_{n}\right)$ for which $\varphi\left(x_{n}\right)$ is bounded in $\mathbb{R}$ and $\varphi^{\prime}\left(x_{n}\right) \rightarrow 0$ (as $\left.n \rightarrow \infty\right)$ possesses a convergent subsequence.

To prove the main result, we will use the following sadde point theorem due to Rabinowitz [11] (or see [8]).

Theorem 2. Let $\varphi \in C^{1}(H, \mathbb{R})$ and $H=H^{-} \oplus H^{+}, \operatorname{dim}\left(H^{-}\right)<\infty$, $\operatorname{dim}\left(H^{+}\right)=\infty$. We suppose that:

(a) There exist a bounded neighborhood 0 of 0 in $H^{-}$and a constant $\alpha$ such that $\left.\varphi\right|_{\partial D} \leqslant \alpha$

(b) there exists a constant $\beta>\alpha$ such that $\left.\varphi\right|_{H^{+}} \geqslant \beta$;

(c) $\varphi$ satisfies (PS).

Then functional $\varphi$ has a critical point in $H$.

\section{The prof of Theorem 1}

In this section, we first show that the functional $\varphi$ satisfies the Palais-Smale condition.

Lemma 2. Assume that the conditions $\left(H_{1}\right),\left(H_{2}\right)$ and $\left(H_{3}\right)$ hold. Then $\varphi$ defined by (2.1) satisfies (PS).

Proof. Let $M>0$ be a constant and $\left\{x_{n}\right\} \subset H$ be a sequence satisfying

$$
\begin{aligned}
\left|\varphi\left(x_{n}\right)\right|= & \mid \frac{1}{2} \int_{0}^{2 \pi} x_{n}^{\prime 2} d t-\frac{m^{2}}{2} \int_{0}^{2 \pi} x_{n}^{2} d t-\int_{0}^{2 \pi} F\left(t, x_{n}\right) d t \\
& +\int_{0}^{2 \pi} e(t) x_{n}(t) d t+\sum_{j=1}^{p} \int_{0}^{x_{n}\left(t_{j}\right)} I_{j}(t) d t \mid \leqslant M
\end{aligned}
$$

and

$$
\lim _{n \rightarrow \infty}\left\|\varphi^{\prime}\left(x_{n}\right)\right\|=0 .
$$


We first prove that $\left\{x_{n}\right\}$ is bounded in $H$ by contradiction. Assume that $\left\{x_{n}\right\}$ is unbounded. Let $\left\{z_{k}\right\}$ be an arbitrary sequence bounded in $H$. It follows from (3.2) that, for any $k \in \mathbb{N}$,

$$
\lim _{n \rightarrow \infty}\left|\varphi^{\prime}\left(x_{n}\right) z_{k}\right| \leqslant \lim _{n \rightarrow \infty}\left\|\varphi^{\prime}\left(x_{n}\right)\right\|\left\|z_{k}\right\|=0 .
$$

Thus

$$
\lim _{n \rightarrow \infty} \varphi^{\prime}\left(x_{n}\right) z_{k}=0 \quad \text { uniformly for } k \in \mathbb{N} \text {. }
$$

Hence,

$$
\begin{aligned}
& \lim _{n \rightarrow \infty}\left(\int_{0}^{2 \pi}\left(x_{n}^{\prime} z_{k}^{\prime}-m^{2} x_{n} z_{k}\right) d t-\int_{0}^{2 \pi}\left(f\left(t, x_{n}\right) z_{k}-e(t) z_{k}\right) d t\right. \\
& \left.\quad+\sum_{j=1}^{p} I_{j}\left(x_{n}\left(t_{j}\right)\right) z_{k}\left(t_{j}\right)\right)=0 .
\end{aligned}
$$

By $\left(H_{1}\right)$ and $\left(H_{2}\right)$, we have

$$
\lim _{n \rightarrow \infty}\left(\int_{0}^{2 \pi} \frac{f\left(t, x_{n}\right) z_{k}-e(t) z_{k}}{\left\|x_{n}\right\|} d t-\frac{\sum_{j=1}^{p} I_{j}\left(x_{n}\left(t_{j}\right)\right) z_{k}\left(t_{j}\right)}{\left\|x_{\eta}\right\|}\right)=0 .
$$

From (3.3) and (3.4), we obtain

$$
\lim _{n \rightarrow \infty} \int_{0}^{2 \pi}\left(\frac{x_{n}}{\left\|x_{n}\right\|} z_{k}^{\prime} m^{2} \frac{x_{n}}{\left\|x_{n}\right\|} z_{k}\right) d t=0
$$

Set $y_{n}=x_{n} /\left\|x_{n}\right\|$. Then we have

$$
\lim _{n \rightarrow \infty} \int_{0}^{2 \pi}\left(y_{n}^{\prime} z_{k}^{\prime}-m^{2} y_{n} z_{k}\right) d t=0
$$

and furthermore,

$$
\lim _{\substack{n \rightarrow \infty \\ i \rightarrow \infty}} \int_{0}^{2 \pi}\left[\left(y_{n}-y_{i}\right)^{\prime} z_{k}^{\prime}-m^{2}\left(y_{n}-y_{i}\right) z_{k}\right] d t=0
$$

Replacing $z_{k}$ in (3.6) by $\left(y_{n}-y_{i}\right)$, we get

$$
\lim _{\substack{n \rightarrow \infty \\ i \rightarrow \infty}}\left(\left\|y_{n}-y_{i}\right\|^{2}-\left(m^{2}+1\right)\left\|y_{n}-y_{i}\right\|_{2}^{2}\right)=0
$$

Due to the compact imbedding $H \hookrightarrow L^{2}(0,2 \pi)$, going to a subsequence,

$$
y_{n} \rightarrow y_{0} \quad \text { weakly in } H, \quad y_{n} \rightarrow y_{0} \quad \text { in } L^{2}(0,2 \pi) \text {. }
$$

Therefore,

$$
\lim _{\substack{n \rightarrow \infty \\ i \rightarrow \infty}}\left\|y_{n}-y_{i}\right\|_{2}^{2}=0
$$


Furthermore, we have

$$
\lim _{\substack{n \rightarrow \infty \\ i \rightarrow \infty}}\left\|y_{n}-y_{i}\right\|^{2}=0,
$$

which implies $\left\{y_{n}\right\}$ is Cauchy sequence in $H$. Thus, $y_{n} \rightarrow y_{0}$ in $H$. It follows from (3.5) and the usual regularity argument for ordinary differential equations (see [3, Chapter 4]) that

$$
y_{0}=k_{1} \sin m t+k_{2} \cos m t
$$

where $k_{1}^{2}+k_{2}^{2}=\frac{1}{\left(m^{2}+1\right) \pi}\left(\left\|y_{0}\right\|=1\right)$. (Different subsequences of $\left\{y_{n}\right\}$ correspond with different $k_{1}$ and $k_{2}$.) Write $(3.7)$ as

$$
y_{0}=\frac{1}{\sqrt{\left(m^{2}+1\right) \pi}} \sin (m t+\theta),
$$

where $\theta$ satisfies $\sin \theta=\frac{k_{2}}{\sqrt{k_{1}^{2}+k_{2}^{2}}}$ and $\cos \theta=\frac{k_{1}}{\sqrt{k_{1}^{2}+k_{2}^{2}}}$

Taking $z_{k}=\frac{1}{\sqrt{\left(m^{2}+1\right) \pi}} \sin (m t+\theta)$, we get, for any $n \in \mathbb{N}$,

$$
\int_{0}^{2 \pi}\left(x_{n}^{\prime} z_{k}^{\prime}-m^{2} x_{n} z_{k}\right) d t=0 .
$$

Thus, it follows from (3.3) and (3.8) that

$$
\begin{gathered}
\lim _{n \rightarrow \infty}\left[\int_{0}^{2 \pi}\left(f\left(t, x_{n}\right)-e(t)\right) \frac{1}{\sqrt{\left(m^{2}+1\right) \pi}} \sin (m t+\theta) d t\right. \\
-\sum_{j=1}^{p} I_{j}\left(x_{n}\left(t_{j}\right) \frac{1}{\sqrt{\left(m^{2}+1\right) \pi}} \sin \left(m t_{j}+\theta\right)\right]=0 .
\end{gathered}
$$

By $\left(H_{1}\right)$ and $\left(H_{2}\right)$, we obtain

$$
\begin{gathered}
\lim _{n \rightarrow \infty}\left[\int_{0}^{2 \pi}\left(f\left(t, x_{n}\right)-e(t)\right)\left(\frac{1}{\sqrt{\left(m^{2}+1\right) \pi}} \sin (m t+\theta)-y_{n}\right) d t\right. \\
\left.-\sum_{j=1}^{p} I_{j}\left(x_{n}\left(t_{j}\right)\right)\left(\frac{1}{\sqrt{\left(m^{2}+1\right) \pi}} \sin \left(m t_{j}+\theta\right)-y_{n}\left(t_{j}\right)\right)\right]=0 .
\end{gathered}
$$

It follows from (3.9) and (3.10) that

$$
\lim _{n \rightarrow \infty}\left[\int_{0}^{2 \pi}\left(f\left(t, x_{n}\right)-e(t)\right) y_{n} d t-\sum_{j=1}^{p} I_{j}\left(x_{n}\left(t_{j}\right)\right) y_{n}\left(t_{j}\right)\right]=0 .
$$

Hence, replacing $z_{k}$ in (3.3) by $y_{n}$, we have

$$
\lim _{n \rightarrow \infty} \int_{0}^{2 \pi}\left(x_{n}^{\prime} \frac{x_{n}^{\prime}}{\left\|x_{n}\right\|}-m^{2} x_{n} \frac{x_{n}}{\left\|x_{n}\right\|}\right) d t=0 .
$$


Now, dividing (3.1) by $\left\|x_{n}\right\|$, we get

$$
\begin{aligned}
& \left|\frac{1}{2} \int_{0}^{2 \pi}\left(\frac{x_{n}^{2}}{\left\|x_{n}\right\|}-\frac{m^{2} x_{n}^{2}}{\left\|x_{n}\right\|}\right) d t-\int_{0}^{2 \pi} \frac{F\left(t, x_{n}\right)-e(t) x_{n}}{\left\|x_{n}\right\|}\right| \\
& \quad \leqslant \frac{M}{\left\|x_{n}\right\|}+\frac{\left|\sum_{j=1}^{p} \int_{0}^{x_{n}\left(t_{j}\right)} I_{j}(t) d t\right|}{\left\|x_{n}\right\|} \leqslant \frac{M}{\left\|x_{n}\right\|}+\sum_{j=1}^{p} c_{j} \frac{\left|x_{n}\left(t_{j}\right)\right|}{\left\|x_{n}\right\|} .
\end{aligned}
$$

Note that $\frac{x_{n}}{\left\|x_{n}\right\|} \rightarrow \frac{1}{\sqrt{\left(m^{2}+1\right) \pi}} \sin (m t+\theta)$ in $H$. Hence, from (3.11) and (3.12), we have

$$
\begin{aligned}
& \liminf _{n \rightarrow \infty} \int_{0}^{2 \pi} \frac{F\left(t, x_{n}\right)-e(t) x_{n}}{\left\|x_{n}\right\|} d t \leqslant \liminf _{n \rightarrow \infty}\left(\frac{M}{\left\|x_{n}\right\|}+\sum_{j=1}^{p} c_{j} \frac{\mid x_{n}\left(t_{j}\right)}{\| x_{n} \mid}\right) \\
& =\sum_{j=1}^{p} c_{j}\left|\frac{1}{\sqrt{\left(m^{2}+1\right) \pi}} \sin \left(m t_{j}+\theta\right)\right|=\frac{1}{\sqrt{\left(m^{2}+1\right) \pi}} \sum_{j=1}^{p} c_{j} \mid \sin \left(n n t_{j}+\theta\right)
\end{aligned}
$$

Due to the compact imbedding $H \hookrightarrow C(0,2 \pi)$, we have $\frac{x_{n}}{\left\|x_{n}\right\|} \sqrt{\frac{1}{\sqrt{\left(m^{2}+1\right) \pi}}} \times$ $\sin (m t+\theta)$ in $C(0,2 \pi)$. Furthermore,

where

$$
\lim _{n \rightarrow \infty} x_{n}(t)=\left\{\begin{array}{l}
+\infty, \begin{array}{l}
\forall t \in I \\
-\infty
\end{array}, \forall t \in I
\end{array},\right.
$$

$$
I_{+}:=\{t \in[0,2 \pi] \mid \sin (m t+\theta)>0\}, \quad I_{-}:=\{t \in[0,2 \pi] \mid \sin (m t+\theta)<0\} .
$$

Using Fatou's lemma, we get

$$
\begin{aligned}
& \liminf _{n \rightarrow \infty} \int_{0}^{2 \pi} \frac{F\left(t, x_{n}\right)}{\left\|x_{n}\right\|} d t \\
& =\liminf _{n \rightarrow \infty}\left[\int_{I_{+}} \frac{F\left(t, x_{n}\right)}{x_{n}} \frac{x_{n}}{\left\|x_{n}\right\|} d t-\int_{I_{-}} \frac{F\left(t, x_{n}\right)}{x_{n}} \frac{-x_{n}}{\left\|x_{n}\right\|} d t\right]
\end{aligned}
$$$$
\begin{aligned}
& \text { Thus, by a simple computation, we have } \\
& \operatorname{limini}_{n \rightarrow \infty} \frac{F\left(t, x_{n}\right)}{x_{n}} \frac{x_{n}}{\left\|x_{n}\right\|} d t-\int_{I_{-}} \limsup _{n \rightarrow \infty} \frac{F\left(t, x_{n}\right)}{x_{n}} \frac{-x_{n}}{\left\|x_{n}\right\|} d t \text {. }
\end{aligned}
$$

$$
\begin{aligned}
& \liminf _{n \rightarrow \infty} \int_{0}^{2 \pi} \frac{F\left(t, x_{n}\right)}{\left\|x_{n}\right\|} d t \\
& \geqslant \frac{1}{\sqrt{\left(m^{2}+1\right) \pi}} \int_{0}^{2 \pi}\left[F_{+}(t) \sin ^{+}(m t+\theta)-F_{-}(t) \sin ^{-}(m t+\theta)\right] d t .
\end{aligned}
$$

Hence, it follows from (3.13) and (3.14) that

$$
\begin{aligned}
& \sum_{j=1}^{p} c_{j}\left|\sin \left(m t_{j}+\theta\right)\right|+\int_{0}^{2 \pi} e(t) \sin (m t+\theta) d t \\
& \quad \geqslant \int_{0}^{2 \pi}\left[F_{+}(t) \sin ^{+}(m t+\theta)-F_{-}(t) \sin ^{-}(m t+\theta)\right] d t .
\end{aligned}
$$


This contradicts $\left(H_{3}\right)$. It implies that the sequence $\left(x_{n}\right)$ is bounded. Thus, there exists $x_{0} \in H$ such that $x_{n} \rightarrow x_{0}$ weakly in $H$. Due to the compact imbedding $H \hookrightarrow L^{2}(0,2 \pi)$ and $H \hookrightarrow C(0,2 \pi)$, going to a subsequence,

$$
x_{n} \rightarrow x_{0} \quad \text { in } L^{2}(0,2 \pi), \quad x_{n} \rightarrow x_{0} \quad \text { in } C(0,2 \pi) .
$$

From (3.3), we obtain

$$
\lim _{\substack{n \rightarrow \infty \\ i \rightarrow \infty}}\left(\int_{0}^{2 \pi}\left(\left(x_{n}^{\prime}-x_{i}^{\prime}\right) z_{k}^{\prime}-m^{2}\left(x_{n}-x_{i}\right) z_{k}\right) d t-\int_{0}^{2 \pi}\left(f\left(t, x_{n}\right)-f\left(t, x_{i}\right)\right) z_{k} d t\right.
$$

$$
\left.+\sum_{j=1}^{p}\left(I_{j}\left(x_{n}\left(t_{j}\right)\right)-I_{j}\left(x_{i}\left(t_{j}\right)\right)\right) z_{k}\left(t_{j}\right)\right)=0 .
$$

Replacing $z_{k}$ by $x_{n}-x_{i}$ in above equality, we get

$$
\begin{aligned}
& \lim _{\substack{n \rightarrow \infty \\
i \rightarrow \infty}}\left(\int_{0}^{2 \pi}\left(\left(x_{n}^{\prime}-x_{i}^{\prime}\right)^{2}-m^{2}\left(x_{n}-x_{i}\right)^{2}\right) d t\right. \\
& \quad-\int_{0}^{2 \pi}\left(f\left(t, x_{n}\right)-f\left(t, x_{i}\right)\right)\left(x_{n}-x_{i}\right) d t \\
& \left.\quad+\sum_{j=1}^{p}\left(I_{j}\left(x_{n}\left(t_{j}\right)\right)-I_{j}\left(x_{i}\left(t_{j}\right)\right)\right)\left(x_{n}\left(t_{j}\right)-x_{j}\left(t_{j}\right)\right)\right)=0 .
\end{aligned}
$$

By $\left(H_{1}\right)$ and $\left(H_{2}\right)$, we have

$$
\begin{aligned}
& \lim _{\substack{n \rightarrow \infty \\
i \rightarrow \infty}} \int_{0}^{2 \pi}\left(f(t, x)-f\left(t, x_{i}\right)\right)\left(x_{n}-x_{i}\right) d t=0 \\
& \left.\lim _{\substack{n \rightarrow \infty \\
i \rightarrow \infty}} \sum_{j=1}^{p} I_{j}\left(x_{n}\left(t_{j}\right)\right)-I_{j}\left(x_{i}\left(t_{j}\right)\right)\right)\left(x_{n}\left(t_{j}\right)-x_{i}\left(t_{j}\right)\right)=0 .
\end{aligned}
$$

Thus, it follows from (3.15), (3.16) and (3.17) that

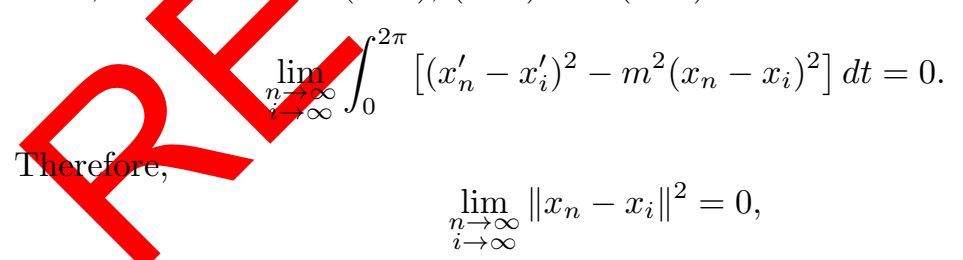

which implies $x_{n} \rightarrow x_{0}$ in $H$. It shows that $\varphi$ satisfies (PS).

Now, we can give the proof of Theorem 1.

Proof of Theorem 1. Denote $H^{+}=\operatorname{span}\{\sin (m+1) t, \cos (m+1) t, \ldots\}$ and $H^{-}=\mathbb{R} \oplus \operatorname{span}\{\sin t, \cos t, \sin 2 t, \cos 2 t, \ldots, \sin m t, \cos m t\}$.

We first prove that

$$
\liminf _{\|x\| \rightarrow \infty} \varphi(x)=-\infty, \quad \text { for } x \in H^{-}
$$


by contradiction. Assume that there exists a sequence $\left(x_{n}\right) \subset H^{-}$such that $\left\|x_{n}\right\| \rightarrow \infty($ as $n \rightarrow \infty)$ and there exists a constant $c_{-}$satisfying

$$
\liminf _{n \rightarrow \infty} \varphi\left(x_{n}\right) \geqslant c_{-} .
$$

By $\left(H_{1}\right)$, we have

$$
\lim _{n \rightarrow \infty} \int_{0}^{2 \pi} \frac{F\left(t, x_{n}\right)-e(t) x_{n}}{\left\|x_{n}\right\|^{2}} d t=0
$$

By $\left(H_{2}\right)$, we get

$$
\lim _{n \rightarrow \infty} \sum_{j=1}^{p} \frac{\int_{0}^{x_{n}\left(t_{j}\right)} I_{j}(t) d t}{\left\|x_{n}\right\|^{2}}=0
$$

From (3.19) and the definition of $\varphi$, we obtain

$$
\begin{aligned}
& \liminf _{n \rightarrow \infty}\left[\frac{1}{2} \int_{0}^{2 \pi} \frac{x_{n}^{\prime 2}-m^{2} x_{n}^{2}}{\left\|x_{n}\right\|^{2}} d t-\int_{0}^{2 \pi} \frac{F\left(t, x_{n}\right)-e(t) x_{n}}{\left\|x_{n}\right\|^{2}} d t+\sum_{j=1}^{p} \frac{v_{0}^{x_{n}}\left(t_{j}\right)}{\left\|x_{n}\right\|^{2}}\right] d t \\
& \quad \geqslant 0 .
\end{aligned}
$$

By the definition of $H^{-}$, we have, for $x \in H^{-}$,

$$
\int_{0}^{2 \pi}\left(x^{\prime 2}-m^{2} x^{2}\right) d t=\|x\|^{2}-\left(m^{2}+1\right)\|x\|_{2}^{2} \leqslant 0 .
$$

The equality in (3.23) holds only for

$$
\frac{1}{\sqrt{\left(m^{2}+1\right) \pi}} \sin (m t+\theta), \quad \theta \in \mathbb{R} \text {. }
$$

Set $y_{n}=\frac{x}{\left\|x_{n}\right\|}$. Since $\operatorname{dim} H^{-}<\infty$, going to a subsequence, there exists $y_{0} \in H^{-}$such that $y_{n} \rightarrow y_{0}$ in $H$ and $y_{n} \rightarrow y_{0}$ in $L^{2}(0,2 \pi)$. Then $(3.20)$, (3.21) - (3.22) and (3.23) imply that

$$
y_{0}=\frac{1}{\sqrt{\left(m^{2}+1\right) \pi}} \sin (m t+\theta), \quad \theta \in \mathbb{R} .
$$

By (3.19), we have, for $n$ large enough,

$$
\frac{1}{2} \int_{0}^{2 \pi} \frac{x_{n}^{\prime 2}-m^{2} x_{n}^{2}}{\left\|x_{n}\right\|} d t-\int_{0}^{2 \pi} \frac{F\left(t, x_{n}\right)-e(t) x_{n}}{\left\|x_{n}\right\|} d t+\sum_{j=1}^{p} \frac{\int_{0}^{x_{n}\left(t_{j}\right)} I_{j}(t) d t}{\left\|x_{n}\right\|} \geqslant \frac{c_{-}}{\left\|x_{n}\right\|} .
$$

It follows from $x_{n} \in H^{-}$that

$$
\int_{0}^{2 \pi} \frac{x_{n}^{\prime 2}-m^{2} x_{n}^{2}}{\left\|x_{n}\right\|} \leqslant 0
$$


From (3.24), (3.25) and $\left(H_{2}\right)$, we get, for $n$ large enough,

$$
\begin{aligned}
\frac{c_{-}}{\left\|x_{n}\right\|} & \leqslant-\int_{0}^{2 \pi} \frac{F\left(t, x_{n}\right)-e(t) x_{n}}{\left\|x_{n}\right\|} d t+\sum_{j=1}^{p} \frac{\int_{0}^{x_{n}\left(t_{j}\right)} I_{j}(t) d t}{\left\|x_{n}\right\|} \\
& \leqslant-\int_{0}^{2 \pi} \frac{F\left(t, x_{n}\right)-e(t) x_{n}}{\left\|x_{n}\right\|} d t+\sum_{j=1}^{p} c_{j} \frac{\left|x_{n}\left(t_{j}\right)\right|}{\left\|x_{n}\right\|} .
\end{aligned}
$$

Thus,

$$
\begin{gathered}
\liminf _{n \rightarrow \infty} \int_{0}^{2 \pi}\left(\frac{F\left(t, x_{n}\right)}{x_{n}}-e(t)\right) \frac{x_{n}}{\left\|x_{n}\right\|} d t \leqslant \liminf _{n \rightarrow \infty} \sum_{j=1}^{p} c_{j} \frac{\left|x_{n}\left(t_{j}\right)\right|}{\left\|x_{n}\right\|} \\
=\sum_{j=1}^{p} c_{j} \frac{1}{\sqrt{\left(m^{2}+1\right) \pi}}\left|\sin \left(m t_{j}+\theta\right)\right|=\frac{1}{\sqrt{\left(m^{2}+1\right) \pi}} \sum_{j=1}^{p} c_{j} \mid \sin (m t
\end{gathered}
$$

Using a argument similarly as in the proof of Lemma 2, we get

$$
\begin{aligned}
& \sum_{j=1}^{p} c_{j}\left|\sin \left(m t_{j}+\theta\right)\right|+\int_{0}^{2 \pi} e(t) \sin (m t+\theta) d t \\
& \quad \geqslant \int_{0}^{2 \pi}\left(F_{+}(t) \sin ^{+}(m t+\theta)-F_{-}(t) \sin ^{-}(m t+\theta)\right) d t,
\end{aligned}
$$

which is a contradiction to $\left(H_{3}\right)$. Then $(3.18)$ holds.

Next, we prove that

$$
\lim _{x \|} \varphi(x)=\infty, \text { for all } x \in H^{+} \text {, }
$$

and $\varphi$ is bounded on bounded sets.

Because of the compact imbedding of $H \hookrightarrow C(0,2 \pi)$ and $H \hookrightarrow L^{2}(0,2 \pi)$, there exists constants $m_{1}, m_{2}$ such that

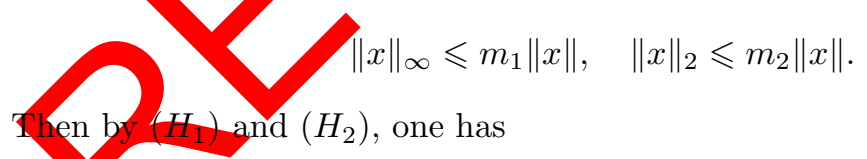

$$
\begin{aligned}
|\varphi(x)| & =\mid \frac{1}{2} \int_{0}^{2 \pi} x^{\prime 2} d t-\frac{m^{2}}{2} \int_{0}^{2 \pi} x^{2} d t-\int_{0}^{2 \pi}[F(t, x)-e(t) x] d t \\
& +\sum_{j=1}^{p} \int_{0}^{x\left(t_{j}\right)} I_{j}(t) d t \mid \\
\leqslant & \frac{1}{2}\|x\|^{2}+\frac{m^{2}}{2} m_{2}^{2}\|x\|^{2}+\int_{0}^{2 \pi}(|p(t)||x|+|e(t)||x|) d t+\sum_{j=1}^{p} c_{j}\left|x\left(t_{j}\right)\right| \\
\leqslant & \frac{1+m^{2} m_{2}^{2}}{2}\|x\|^{2}+m_{1}\left(\|p\|_{1}+\|e\|_{1}\right)\|x\|+\sum_{j=1}^{p} c_{j} m_{1}\|x\| .
\end{aligned}
$$


Hence, $\varphi$ is bounded on bounded sets of $H$.

By the definition of $H^{+}$, we have, for $x \in H^{+}$,

$$
\|x\|^{2} \geqslant\left((m+1)^{2}+1\right)\|x\|_{2}^{2} .
$$

Thus, from (3.26) and (3.27), we obtain

$$
\begin{aligned}
\varphi(x)= & \frac{1}{2} \int_{0}^{2 \pi} x^{\prime 2} d t-\frac{m^{2}}{2} \int_{0}^{2 \pi} x^{2} d t-\int_{0}^{2 \pi}[F(t, x)-e(t) x] d t \\
& +\sum_{j=1}^{p} \int_{0}^{x\left(t_{j}\right)} I_{j}(t) d t \\
\geqslant & \frac{2 m+1}{2\left((m+1)^{2}+1\right)}\|x\|^{2}-m_{1}\left(\|p\|_{1}+\|e\|_{1}+\sum_{j=1}^{p} c_{j}\right)\|x\|, \quad \text { for all } x \in H^{+}
\end{aligned}
$$

which implies

Up to now, the conditions $(a)$ and $(b)$ of Theorem 2 are satisfied. According to Lemma 2, $(c)$ is also satisfied. Hence, by Theorem 2, Eq. (1.1) has at least one solution. This completes the proof.

\section{Conclusions}

A generalized Landesman Lazer type condition for the existence of periodic solutions of second order impulsive differential equations at resonance was obtained.

\section{Acknowledgment}

The authors would like to express their thanks to the editor of the journal and the anonynous referees for their carefully reading of the first draft of the manuscript and making many helpful comments and suggestions which improved the presentation of the paper.

\section{References}

[1] I. Bogun. Existence of weak solutions for impulsive p-Laplacian problem with superlinear impulses. Nonlinear Anal. Real World Appl., 13(6):2701-2707, 2012. http://dx.doi.org/10.1016/j.nonrwa.2012.03.014.

[2] W. Ding and D. Qian. Periodic solutions for sublinear systems via variational approach. Nonlinear Anal. Real World Appl., 11(4):2603-2609, 2010. http://dx.doi.org/10.1016/j.nonrwa.2009.09.007.

[3] S. Fučík. Solvability of Nonlinear Equations and Boundary Value Problems. D. Reidel Publ. Company, Holland, 1980.

[4] V. Lakshmikantham, D.D. Bainov and P.S. Simeonov. Theory of Impulsive Differential Equations. World Scientific, Singapore, 1989. 
[5] E.M. Landesman and A.C. Lazer. Nonlinear perturbations of linear elliptic boundary value problems at resonance. J. Math. Mech., 19:609-623, 1970.

[6] A.C. Lazer. On schauder's fixed point theorem and forced second order nonlinear oscillations. J. Math. Anal. Appl., 21:421-425, 1968.

http://dx.doi.org/10.1016/0022-247X(68)90225-4.

[7] A.C. Lazer and D.E. Leach. Bounded perturbations of forced harmonic oscillators at resonance. Ann. Mat. Pura Appl., 82(4):49-68, 1969.

http://dx.doi.org/10.1007/BF02410787.

[8] J. Mawhin and M. Willem. Critical Point Theory and Hamiltonian Systems. Springer-Verlag, Berlin, 1989.

[9] J.J. Nieto and D. O'Regan. Variational approach to impulsive tions. Nonlinear Anal. Real World Appl., 10(2):680-690, 2009. http://dx.doi.org/10.1016/j.nonrwa.2007.10.022.

[10] D. Qian and X. Li. Periodic solutions for ordinary differentialyequations with sublinear impulsive effects. J. Math. Anal. Appl., 303:288-303, 2005, http://dx.doi.org/10.1016/j.jmaa.2004.08.034.

[11] P.H. Rabinowitz. Minmax Methods in Critical Point Theory with Applications to Differential Equations, volume 65 of CBMS Reg. Conf. Ser. in Math. Amer. Math. Soc., Providence, RI, 1986.

[12] H. Sun, Y. Li, J.J. Nieto and Q. Tang. Existence of solutions for Sturm-Liouville boundary value problem of impulsive differential equations. Abstr. Appl. Anal., 2012:Article ID 707163, 19 pages, 2012. http://dx.doi.org/10.1155/2012/707163.

[13] J. Sun, J. Chu and H. Chen. Periodic solution generated by impulses for singular differential equations. J. Math. Anal. Appl., 404:562-569, 2013. http://dx.doi.org/101016/j.jmaa.2013.03.036.

[14] Y. Tian and W/Ge. Applications of variational methods to boundary value problem for impulsive differential equations. Proc. Edinb. Math. Soc., 2:509$527,2008$.

[15] P. Tomiczek. The duffing equation with the potential landesman-lazer condition. Nonlinear Anal., 70(2):735-740, 2009.

http://dx.doi.org/10.1016/j.na.2008.01.006.

[16] J. Xiao, J. Nieto and Z. Luo. Multiplicity of solutions for nonlinear second order impulsive differential equations with linear derivative dependence via Iationalmethods. Commun. Nonlinear Sci. Numer. Simul., 1:426-432, 2012. http://dx.doi.org/10.1016/j.cnsns.2011.05.015.

[17] H. Zhang and Z. Li. Variational approach to impulsive differential equations with periodic boundary conditions. Nonlinear Anal. Real World Appl., 11(1):67-78, 2010. http://dx.doi.org/10.1016/j.nonrwa.2008.10.016.

[18] X. Zhang and Q. Meng. Nontrivial periodic solutions for delay differential systems via Morse theory. Nonlinear Anal., 74(5):1960-1968, 2011. http://dx.doi.org/10.1016/j.na.2010.11.003.

[19] Z. Zhang and R. Yuan. An application of variational methods to Dirichlet boundary value problem with impulses. Nonlinear Anal. Real World Appl., 11(1):155162, 2010. http://dx.doi.org/10.1016/j.nonrwa.2008.10.044. 